\title{
Micronutrientes catiônicos: fontes, doses e ambientes
}

\author{
Regina Maria Quintão Lana' ${ }^{1}$, Adriane de Andrade Silva², Bruno Nicchio³, \\ Joicy Vitória Miranda Peixoto ${ }^{3}$, Emmerson Rodrigues de Moraes ${ }^{4}$
}

\footnotetext{
${ }^{1}$ Universidade Federal de Uberlândia, Instituto de Ciências Agrárias, Av Amazonas, s/n, Bloco 4C - Sala 112, Umuarama, CEP 38400-734, Uberlândia-MG, Brasil. Caixa Postal 593. E-mail: rmqlana@iciag.ufu.br

${ }^{2}$ Universidade Federal de Uberlândia, Instituto de Ciências Agrárias, Rua Goiás, 2000, Vila Nova, CEP 38500-000, Monte Carmelo-MG, Brasil. E-mail: zoodrika@uol.com.br

${ }^{3}$ Universidade Federal de Uberlândia, Programa de Pós-Graduação em Agronomia, Av Pará, 1720, Umuarama, CEP 38408-100, Uberlândia-MG, Brasil. E-mail: bruno_nicchio@hotmail.com; joicymmeixoto@yahoo.com.br

${ }^{4}$ Universidade Federal de Goiás, Programa de Pós-Graduação em Agronomia, Escola de Agronomia, Avenida Esperança, s/n, Campus Samambaia, CEP 74690-900, Goiânia-GO, Brasil. E-mail: emmersonagro@yahoo.com.br
}

\section{RESUMO}

Objetivou-se com este trabalho caracterizar a disponibilidade de $\mathrm{Cu}, \mathrm{Mn}$ e Zn em quatro Oxisols in the Triângulo Mineiro, utilizando-se fertilizantes na forma de quelatos de EDTA, óxido e sulfato. O delineamento experimental foi inteiramente casualizado em esquema fatorial $(3 \times 8)$, sendo a combinação de três fontes (quelatos de EDTA, e nas formas de óxido e de sulfato) em oito concentrações (10, $20,40,60,80,100,120$ e $140 \mathrm{mg} \mathrm{L}^{-1}$ de micronutriente), com três repetições em quatro tipos de solo. 0 uso de fertilizante a base de quelato de EDTA proporciona aumento nos teores de $\mathrm{Cu}$ (cobre) e Zn (zinco) nos solos avaliados (Latossolo Amarelo textura muito argilosa - cultivo de soja; Latossolo Vermelho textura muito argilosa - cerrado nativo e Latossolo Amarelo textura média - cultivo de milho). Nas amostras do Latossolo Vermelho de textura muito argilosa (Latossolo Vermelho textura muito argilosa - cerrado nativo) a disponibilidade de Cu aumenta linearmente em função da aplicação de fertilizante quelatado (EDTA). O fertilizante quelatado (EDTA) aumenta linearmente de maneira mais acentuada os teores de $\mathrm{Zn}$ nas amostras do solo Latossolo Amarelo textura muito argilosa e Latossolo Amarelo de textura média. Em geral, a aplicação de fertilizante quelatado (EDTA) proporciona incrementos nos níveis de Cu e Zn.

Palavras-chave: cosmoquel-EDTA; fertilizantes; micronutrientes

\section{Cationic micronutrients: sources, doses and environments}

\begin{abstract}
The objective of this work was to characterize the availability of $\mathrm{Cu}, \mathrm{Mn}$ and $\mathrm{Zn}$ in four Latosols Triângulo Mineiro, using fertilizers in the form of EDTA chelates, oxides and sulfate. The experimental design was completely randomized in a factorial arrangement $(3 \times 8)$, and the combination of three sources (chelates EDTA, and in the forms of oxide and sulphate) in eight concentrations $\left(10,20,40,60,80,100,120\right.$ and $140 \mathrm{mg} \mathrm{L}^{-1}$ micronutrient) with three replications in each soil. The fertilizer EDTA chelate is more efficient in the availability of $\mathrm{Cu}$ and $\mathrm{Zn}$ compared to other tested sources (liquid and sulfated source), the evaluated soils. The use of fertilizer EDTA chelate base provides an increase in Cu concentration (copper) and Zn (zinc) in the evaluated soils (Yellow Oxisol medium texture - soy cultivation and Yellow Oxisol medium texture - maize crop). Samples from the very clayey Oxisol (native savannah) the availability of $\mathrm{Cu}$ increases linearly as a function of the application of savannah fertilizer (EDTA). The chelated fertilizer (EDTA) increases linearly more sharply Zn levels in soil samples Oxisol clayey and Oxisol of medium texture. In general, the application of fertilizer chelated (EDTA) provides increases in levels of $\mathrm{Cu}$ and $\mathrm{Zn}$.
\end{abstract}

Key words: cosmoquel-EDTA; fertilizers; micronutrients 


\section{Introdução}

A região do cerrado apresenta solos originalmente muito intemperizados e após a abertura das fronteiras agrícolas, uma grande demanda de micronutrientes têm-se demonstrado. Especialmente com o aumento de produtividade dessas áreas e as constantes exportações destes micronutrientes, tornou-se mais comum o relato de sintomas de deficiência e resposta em produtividade com a aplicação de fontes de micronutrientes (Silva et al., 2014). No entanto, muitas vezes, a adição de fertilizantes específicos, como no caso dos micronutrientes tem sido negligenciada, resultado da resistência dos produtores em aplicar fontes de micronutrientes (como os sulfatos e óxidos), em solos com baixa disponibilidade e que não respondem às exigências das culturas, o que pode limitar sua produção (Carvalho et al., 2012; Udeigwe et al., 2016).

Além disso, a característica químico-física dos solos interfere na disponibilidade dos micronutrientes o que faz necessário o conhecimento dos fatores que afetam os processos de disponibilidade, como $\mathrm{pH}$ do solo, teor de matéria orgânica, textura, óxidos de ferro e alumínio e interações com outros micronutrientes (Marschner, 1995; Gonçalves et al., 2013; Udeigwe et al., 2016).

Conforme Carvalho et al. 2012, os micronutrientes, cobre $(\mathrm{Cu})$, manganês $(\mathrm{Mn})$ e zinco $(\mathrm{Zn})$ são muito importantes no desenvolvimento e à sobrevivência dos vegetais, participando direta ou indiretamente de diversas atividades metabólicas, tendo como fonte primária o solo, de onde são absorvidos pelas plantas. Por isso, o manejo adequado e eficiente de fertilizantes e micronutrientes na agricultura pode contribuir sensivelmente para o aumento da produção e da produtividade das culturas (Joris et al., 2012; Lopes et al., 2014; Mclaughlin et al., 2013).

Algumas fontes de micronutrientes assim como as fontes quelatadas proporcionam uma ligação mais forte com os nutrientes, como uma proteção da interação química como exemplo, EDTA, DTPA, IDHA, EDDHA, HBED, orgânicos, entre outros (Mclaughlin et al., 2013). Fontes de micronutrientes como as sulfatadas para disponibilizar o nutriente ao solo, dependem dos teores de micronutrientes, dos tipos de argilominerais e dos óxidos presentes no solo (Peak et al., 1999). Para fontes na forma de óxido, diluída em solução aquosa, as características de ligações e polaridades das cargas não têm um comportamento ideal tendendo a ser de mais difícil explicação (López-Rayo et al., 2012).

Segundo Costa (2008), existe carência de trabalhos de pesquisa no Brasil avaliando a eficiência de quelatos ou complexos, principalmente quando aplicados no solo. Assim, a compreensão das interações que ocorrem no sistema soloplanta-água é o que define a capacidade das plantas em responder aos nutrientes adicionados no solo (Alleoni et al., 2009), demonstrando a necessidade de se avaliar o efeito de micronutrientes para aumento da produtividade. Com isso, o aparecimento de novas tecnologias e fontes de micronutrientes têm grande importância para o agronegócio (López-Rayo et al., 2013).

Portanto, objetivou-se com este estudo caracterizar a disponibilidade de $\mathrm{Cu}, \mathrm{Mn}$ e $\mathrm{Zn}$ em quatro Latossolos do Triângulo Mineiro, utilizando-se fertilizantes na forma de quelatos de EDTA, óxido e sulfato.

\section{Material e Métodos}

O experimento foi realizado no Laboratório de Análises de Fertilizantes da Universidade Federal de Uberlândia (LAFER/ UFU), em Uberlândia, Minas Gerais. $\mathrm{O}$ delineamento experimental utilizado foi o inteiramente casualizado (DIC), em esquema fatorial $(3 \times 8)$, com três repetições. O primeiro fator correspondeu as formas das fontes de micronutrientes (quelato, óxido e sulfato) e o segundo, as concentrações aplicadas (concentrações de 10, 20, 40, 60, 80, 100, 120 e 140 $\mathrm{mg} \mathrm{L}^{-1}$ de micronutriente) em amostras de solos com diferentes cultivos agrícolas.

Para avaliar a disponibilidade dos micronutrientes $\mathrm{Cu}, \mathrm{Mn}$ e $\mathrm{Zn}$ foram analisadas curvas de adsorção dos micronutrientes nas amostras de solo coletadas, determinadas pela adição de $20 \mathrm{~mL}$ de uma solução que continham as concentrações de micronutriente, na forma de: quelatos de EDTA (CosmoquelEDTA), fertilizante na forma de óxido de $\mathrm{Cu}, \mathrm{Mn}$ e $\mathrm{Zn}$ e sulfato de $\mathrm{Cu}, \mathrm{Mn}$ e $\mathrm{Zn}$; em $0,01 \mathrm{~mol} \mathrm{~L}^{-1}$ de $\mathrm{CaCl}_{2} 2 \mathrm{H}_{2} \mathrm{O}$ aplicados a 2,0 g de solo seco ao ar, seguindo a metodologia relatada por Soares (2004). As soluções tiveram seus valores de $\mathrm{pH}$ corrigidos para 5,5 imediatamente antes do início do experimento. As suspensões solo-solução foram agitadas por 30 minutos a $220 \mathrm{rpm}$ e, em seguida, centrifugadas a $2000 \mathrm{rpm}$ por 10 minutos, filtradas e analisadas, para o $\mathrm{Cu}, \mathrm{Mn}$ e $\mathrm{Zn}$, por espectrofotometria de absorção atômica.

As concentrações de $\mathrm{Cu}, \mathrm{Mn}$ e $\mathrm{Zn}$ remanescentes foram determinadas após o período de equilíbrio com $\mathrm{CaCl}_{2}(0,01$ mol L-1), ao multiplicar o resultado do espectrofotômetro pela diluição efetuada para cada concentração. As concentrações do micronutriente adsorvido foram consideradas pela diferença entre aquelas inicialmente adicionadas e as concentrações do micronutriente disponível. As concentrações de 10 e $20 \mathrm{mg}$ $\mathrm{dm}^{-3}$ foram realizadas em uma diluição de $20 \mathrm{mg} \mathrm{dm}^{-3}$. As demais concentrações $\left(40,60,80,100,120\right.$ e $\left.140 \mathrm{mg} \mathrm{dm}^{-3}\right)$ foram efetuadas em duas diluições. Na primeira diluição utilizou-se $1 \mathrm{~mL}$ do extrato associado à $3,4,5,6,7$ e $8 \mathrm{~mL}$ de $\mathrm{CaCl}_{2}$, respectivamente, para cada concentração. A segunda diluição foi realizada por meio da adição de $1 \mathrm{~mL}$ do extrato a 4, 5, 6, 7, 8 e $9 \mathrm{~mL}$ de $\mathrm{CaCl}_{2}$, respectivamente, para cada concentração.

Foram utilizadas amostras de quatro tipos de solos pertencentes originalmente ao bioma cerrado, com duas texturas diferentes e dois usos e ocupação no momento da retirada das amostras: 1- Latossolo Amarelo textura muito argilosa (LAtma) com cultivo de soja; 2- Latossolo Amarelo textura média (LAtm) com cultivo de milho; 3- Latossolo Vermelho textura muito argilosa (LVtma) com cerrado nativo típico; e 4- Latossolo Amarelo textura média (LAtm) com cerrado nativo em área de chapadão (Embrapa, 2013). As características químicas encontram-se nas Tabelas 1 e 2 . Todos os solos utilizados no experimento foram coletados na profundidade de $0-10 \mathrm{~cm}$, sendo 25 pontos amostrados em cada área selecionada para composição da amostra composta em uma área de $1 \mathrm{ha}^{-1}$, totalizando 96 amostras para cada solo e cada fonte avaliada.

Com a obtenção dos dados, as médias dos tratamentos foram submetidas à análise variância, e, em caso de significância do teste de F, os dados qualitativos foram comparados pelo teste 
Tabela 1. Características químicas de amostras de solo de um Latossolo Amarelo textura muito argilosa (LAtma), Latossolo Amarelo textura média (LAtm), Latossolo Vermelho textura muito argilosa (LVtma) e Latossolo Amarelo textura média (LAtm), Uberlândia, Minas Gerais.

\begin{tabular}{|c|c|c|c|c|c|c|c|c|c|c|c|c|c|}
\hline \multirow{2}{*}{ Solo } & pH & $\mathbf{P}$ & $\mathbf{S}$ & $\mathbf{K}$ & Al & $\mathbf{C a}$ & $\mathrm{Mg}$ & $\mathbf{H}+\mathbf{A l}$ & SB & $\mathbf{T}$ & $\mathbf{V}$ & $\mathbf{m}$ & MO \\
\hline & $\mathrm{H}_{2} \mathrm{O}$ & \multicolumn{2}{|c|}{$\mathrm{mg} \mathrm{dm}^{-3}$} & \multicolumn{7}{|c|}{$\mathrm{cmol}_{\mathrm{c}} \mathrm{dm}^{-3}$} & \multicolumn{3}{|c|}{$\%$} \\
\hline 1 & 6,3 & 15,7 & 12 & 0,28 & 0,0 & 3,7 & 0,9 & 2,50 & 4,88 & 7,38 & 66 & 0 & 3,3 \\
\hline 2 & 6,5 & 13,9 & 4 & 0,08 & 0,0 & 1,9 & 0,5 & 1,90 & 2,48 & 4,38 & 57 & 0 & 1,3 \\
\hline 3 & 5,5 & 14,6 & 4 & 0,07 & 0,2 & 0,8 & 0,2 & 5,20 & 1,07 & 6,27 & 17 & 16 & 3,2 \\
\hline 4 & 5,1 & 1,6 & 4 & 0,05 & 0,7 & 0,1 & 0,1 & 4,90 & 0,25 & 5,15 & 5 & 74 & 1,8 \\
\hline
\end{tabular}

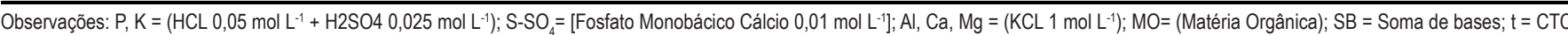
efetiva; $\mathrm{T}=\mathrm{CTC}$ a pH 7,0; $\mathrm{V}=$ Sat. por bases; $\mathrm{m}=$ Sat por Al; Solo 1 = LAtma - cultivo de soja; Solo 2 = LAtm - cultivado de milho; Solo $3=\mathrm{LV}$ tma - cerrado nativo; Solo $4=\mathrm{LAtm}$ - C. $\mathrm{n}$. em chapadão.

Tabela 2. Teores de micronutrientes em amostras de um Latossolo Amarelo textura muito argilosa (LAtma), Latossolo Amarelo textura média (LAtm), Latossolo Vermelho textura muito argilosa (LVtma) e Latossolo Amarelo textura média (LAtm), Uberlândia, Minas Gerais.

\begin{tabular}{cccccc}
\hline \multirow{2}{*}{ Solo $^{1}$} & $\mathbf{B}$ & $\mathbf{C u}$ & $\mathbf{F e}$ & $\mathbf{M n}$ & $\mathbf{Z n}$ \\
\cline { 2 - 6 } & \multicolumn{5}{c}{$\mathbf{~ m g ~ d m}^{-3}$} \\
\hline LAtma - cultivo de soja & 0,21 & 0,8 & 37 & 2,4 & 1,9 \\
LAtm - cultivado de milho & 0,14 & 0,7 & 33 & 2,6 & 1,8 \\
LVtma - cerrado nativo & 0,16 & 1,0 & 98 & 1,4 & 0,4 \\
LAtm - c. n. em chapadão & 0,20 & 0,4 & 280 & 2,6 & 0,2 \\
\hline
\end{tabular}

${ }^{1} \mathrm{Cu}, \mathrm{Mn}$ e $\mathrm{Zn}$ determinados com o extrator DTPA, $\mathrm{B}=[\mathrm{BaCl} 2.2 \mathrm{H} 2 \mathrm{O} 0,0125 \%$ à quente $]$ conforme descrito por Embrapa (2009).

de Tukey e seguido de regressão polinomial para os dados quantitativos, ambos a 5\% de significância com o auxílio do programa estatístico SISVAR (Ferreira, 2008).

\section{Resultados e Discussão}

A interação entre os fatores fontes de micronutrientes e suas concentrações foi significativa para os teores de cobre, manganês e zinco (Tabela 3). Para o LAtm - cerrado nativo em chapadão não foi observada interação entre os teores de $\mathrm{Mn}$ no solo e Cu para o LAtma - cultivo de soja.

O teor disponível de cobre $(\mathrm{Cu})$ foi superior com o uso da fonte EDTA em todas as concentrações, quando comparados com as outras fontes no Latossolo Vermelho textura muito argilosa (LVtma) cerrado nativo e Latossolo Amarelo de textura média (LAtm) cerrado nativo em chapadão (Tabela 4). Esses resultados assemelham-se aos observados por Silva et al. (2014), que encontraram maiores teores de $\mathrm{Cu}$ no solo para a fonte quelatada com EDTA em relação a fonte sulfatada em um Latossolo Amarelo ácrico típico.
A matéria orgânica presente no solo forma complexos com os metais como $\mathrm{Cu}, \mathrm{Mn}$ e $\mathrm{Zn}$, afetando a sua dessorção, mobilidade e solubilidade. Os principais sítios de complexação são os grupamentos carboxílicos e fenólicos (Li et al., 2013; Jalali \& Latifi, 2017). A elevação do $\mathrm{pH}$ a valores acima de 6,0 reduzem a disponibilidade de $\mathrm{Zn}, \mathrm{Cu}$ e $\mathrm{Mn}$. O Zn é fortemente retido em solos argilosos, além disso, os fosfatos tendem a reduzir a sua disponibilidade (Raij, 2011).

Neste experimento, a fonte em forma de óxido e sulfatada pode ter sofrido maior complexação com a matéria orgânica, diferentemente da fonte quelatada que é protegida pelo EDTA. Este fato pode ser justificado pelos atributos do solo que podem influenciar na disponibilidade e adsorção de micronutrientes no solo, pois o $\mathrm{Cu}$ apresenta alta reatividade com os grupos funcionais de partículas orgânicas e pode ser ligado a grupos funcionais de óxidos de Fe e até de Mn ou filossilicatos, uma vez que a matéria orgânica existente no solo e quantidade de argila podem ser os principais responsáveis pela fixação de micronutrientes devido à alta complexidade de sua estrutura, além do $\mathrm{pH}$ e a capacidade de troca iônica (Gonçalves et al., 2013; Brunetto et al., 2014; Udeigwe et al., 2016).

Nas amostras de solo de cerrado nativo (LVtma) o tratamento com óxido entre as concentrações de 100 a 140 $\mathrm{mg} \mathrm{dm}{ }^{-3}$ apresentou menores teores de $\mathrm{Cu}$ (Tabela 4). Os resultados das amostras do Latossolo Amarelo cultivado com milho (LAtm - cultivo de milho), demonstrou que em média o teor de $\mathrm{Cu}$ disponível não variou entre as fontes avaliadas.

Os menores teores de $\mathrm{Cu}$ observado nas concentrações de 100 a $140 \mathrm{mg} \mathrm{dm}^{-3}$ podem estar associados ao teor de argila e a matéria orgânica existente no solo $\left(32 \mathrm{~g} \mathrm{~kg}^{-1}\right)$, além do teor de $\mathrm{Cu}\left(1,0 \mathrm{mg} \mathrm{dm}^{-3}\right)$. A maior disponibilidade de $\mathrm{Cu}$ favoreceu a sua complexação com a matéria orgânica, consequentemente

Tabela 3. Resumo da análise de variância dos teores de Cu Mn e Zn em Latossolos, submetidos a aplicação de diferentes concentrações de micronutrientes usando três tipos de fontes, Uberlândia, Minas Gerais.

\begin{tabular}{|c|c|c|c|c|c|c|}
\hline \multirow{3}{*}{ F.V. } & \multirow{3}{*}{ G.L. } & \multicolumn{5}{|c|}{ Quadrado médio } \\
\hline & & \multicolumn{3}{|c|}{ LVtma - cerrado nativo } & \multicolumn{2}{|c|}{ LAtm - c. n. em chapadão } \\
\hline & & $\mathrm{Cu}$ & Mn & $\mathbf{Z n}$ & $\mathrm{Cu}$ & $\mathrm{Zn}$ \\
\hline Fonte & 2 & $23277,76^{*}$ & $393,04^{*}$ & $439,29^{*}$ & $2376,34^{*}$ & $25,52^{*}$ \\
\hline Dose & 7 & $6018,56^{*}$ & $16801,29^{*}$ & $14065,99^{*}$ & $9353,71^{*}$ & $11896,37^{*}$ \\
\hline$F^{*}$ Dose & 14 & $1191,60^{*}$ & $22,121^{*}$ & $13,59^{*}$ & $528,03^{*}$ & $6,04^{*}$ \\
\hline Resíduo & 46 & 1,10 & 6,90 & 5,04 & 1,85 & 3,17 \\
\hline \multirow[t]{3}{*}{$\mathrm{CV}(\%)$} & & 3,28 & 4,06 & 4,09 & 2,96 & 2,65 \\
\hline & & \multicolumn{3}{|c|}{ LAtm - cultivado de milho } & \multicolumn{2}{|c|}{ LAtma - cultivo de soja } \\
\hline & & $\mathrm{Cu}$ & Mn & Zn & Mn & $\mathrm{Zn}$ \\
\hline Fonte & 2 & $114,08^{*}$ & $270,18^{*}$ & $2808,18^{*}$ & $54,05^{*}$ & $5850,05^{*}$ \\
\hline Dose & 7 & $1764,42^{*}$ & $15753,06^{*}$ & $14295,45^{*}$ & $14234,79^{*}$ & $9122,66^{*}$ \\
\hline$F^{*}$ Dose & 14 & $18,08^{*}$ & $33,99^{*}$ & $68,72^{*}$ & $3,24^{*}$ & $171,88^{*}$ \\
\hline Resíduo & 46 & 1,61 & 10,08 & 2,95 & 1,58 & 1,95 \\
\hline CV $(\%)$ & & 8,33 & 5,17 & 3,18 & 2,18 & 3,35 \\
\hline
\end{tabular}

FV - Fonte de variação; F - Fonte; LVtma - Latossolo Vermelho de textura muito argilosa; LAtm - Latossolo Amarelo de textura média; LAtma - Latossolo Amarelo de textura muito argilosa; Cu - cobre; $\mathrm{Mn}$ - manganês; $\mathrm{Zn}$ - zinco; ${ }^{\text {ns }}$ - não significativo, ${ }^{*}$ - significativo a $5 \%$ pelo teste $\mathrm{F}$. 
Tabela 4. Teores de Cu disponíveis em um Latossolo Vermelho textura muito argilosa (LVtma - cerrado nativo), em um Latossolo Amarelo de textura média (LAtm - cerrado nativo em chapadão) e em um Latossolo Amarelo textura média (LAtm - cultivo de milho) em função de diferentes concentrações de Cu aplicados na forma de quelatos de EDTA, óxido e sulfato, Uberlândia, Minas Gerais.

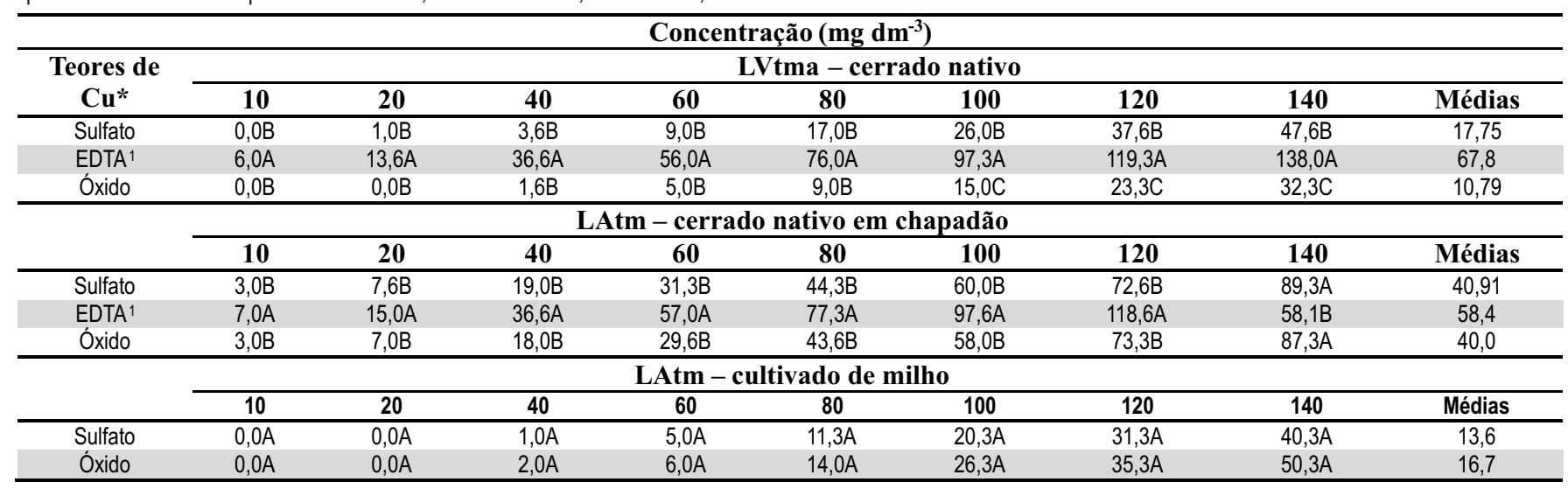

${ }^{1}$ Quelato; *Valores seguidos de mesma letra na coluna não diferem entre si pelo teste de Tukey a $5 \%$ de significância.

reduzindo a sua disponibilidade na solução do solo. A deficiência de $\mathrm{Cu}$ é comum em solos orgânicos. Estes solos mesmo diante de altos teores do nutriente o retêm na forma de complexos estáveis (Raij, 2011). Esses resultados corroboram aos encontrados por Jalali \& Moradi (2013). Estes autores ao avaliarem a sorção dos metais cádmio $(\mathrm{Cd})$, cobre, manganês, níquel $(\mathrm{Ni})$, chumbo $(\mathrm{Pb})$ e zinco em solos calcário observaram que a quantidade adsorvida desses metais foi incrementada conforme o aumento da concentração aplicada. Os autores ao compararem solos com reduzido teor de argila como, por exemplo, solo sob pastagem e solo com alta capacidade de troca catiônica, notificaram menor competição entre os metais no primeiro solo. Eles relataram a seguinte sequência de adsorção $\mathrm{Cu}>\mathrm{Pb}>\mathrm{Cd}>\mathrm{Zn}>\mathrm{Ni}>\mathrm{Mn}$.

$\mathrm{O}$ quelato de EDTA apresentou maior disponibilidade de $\mathrm{Cu}$ no LVtma - cerrado nativo e LAtm - cerrado nativo em chapadão, com aumento linear, assim como as demais fontes, todavia, de maneira mais acentuada (Figura 1a e 1b). O quelato de EDTA apresentou teor máximo de 152,5 e 138,8 $\mathrm{mg} \mathrm{dm}^{-3} \mathrm{de}$ $\mathrm{Cu}$ em ambos os solos (Figura 1a e 1b). Nas amostras de solo cultivadas com milho (LAtm), maiores concentrações de $\mathrm{Cu}$ foram observadas com a fonte na forma de óxido (Figura 1c).

Para os teores de manganês (Mn) no LAtma - cultivo de soja (Tabela 5), não foi observada variação entre as três fontes na concentração de $10 \mathrm{mg} \mathrm{dm}^{-3}$, todavia, na maior concentração $\left(140 \mathrm{mg} \mathrm{dm}^{-3}\right)$ o tratamento sulfato apresentou o menor teor disponível de Mn quando comparado a fonte EDTA. Isso pode ser resultado da possível complexação do micronutriente no solo, devido ao $\mathrm{pH}$ do solo acima de 5,5 e disponibilidade de matéria orgânica (33 $\left.\mathrm{g} \mathrm{kg}^{-1}\right)$ (Gonçalves et al., 2013; Brunetto et al., 2014; Udeigwe et al., 2016). Em comparação com o EDTA, a fonte na forma de óxido, na concentração de $40 \mathrm{mg}$ $\mathrm{dm}^{-3}$ apresentou menores teores de Mn. Essa fonte juntamente com a fonte em forma de sulfato, ambas na concentração de 60 $\mathrm{mg} \mathrm{dm}{ }^{-3}$ apresentaram o menor teor de Mn (Tabela 5).

Não se verificou variação nas menores concentrações das três fontes estudadas no LVtma - cerrado nativo e LAtm cultivo de milho. No entanto, nas maiores concentrações o tratamento quelato de EDTA apresentou menores teores de Mn (Tabela 5). Esses resultados diferem de López-Rayo et al. (2013), que verificaram melhores resultados com aplicação de
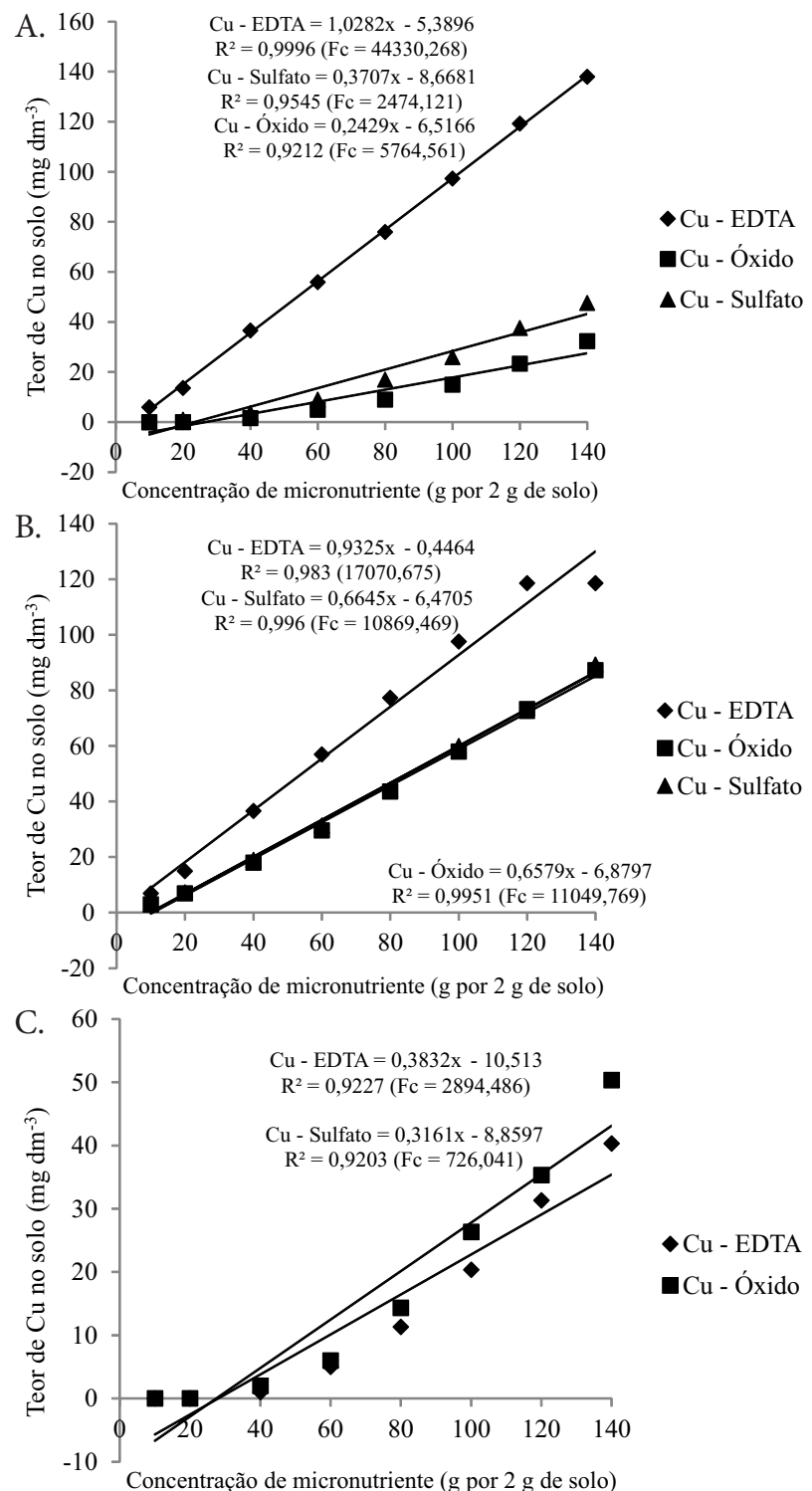

Figura 1. Teores de $\mathrm{Cu}$ em um (a) Latossolo Vermelho de textura muito argilosa (LVtma - cerrado nativo), em um (b) Latossolo Amarelo textura média (LAtm - cerrado nativo em chapadão) e um (c) Latossolo Amarelo textura média (LAtm - cultivo de milho) em função de diferentes concentrações de $\mathrm{Cu}$ aplicados na forma de quelatos de EDTA, óxido e sulfato, Uberlândia, Minas Gerais. 
Tabela 5. Teores de Mn disponíveis em um Latossolo Amarelo textura muito argilosa (LAtma - cultivo de soja), em um Latossolo Vermelho de textura muito argilosa (LVtma - cerrado nativo), e em um Latossolo Amarelo textura média (LAtm - cultivo de milho) em função de diferentes concentrações de Mn aplicados na forma de quelatos de EDTA, óxido e sulfato, Uberlândia, Minas Gerais.

\begin{tabular}{|c|c|c|c|c|c|c|c|c|c|}
\hline \multicolumn{10}{|c|}{ Concentração $\left(\mathrm{mg} \mathrm{dm}^{-3}\right)$} \\
\hline \multirow{2}{*}{$\begin{array}{c}\text { Teores } \\
\text { de Mn* }\end{array}$} & \multicolumn{9}{|c|}{ LAtma - cultivo de soja } \\
\hline & 10 & 20 & 40 & 60 & 80 & 100 & 120 & 140 & Médias \\
\hline Sulfato & $7,0 \mathrm{~A}$ & $13,3 \mathrm{C}$ & $30,0 A B$ & $46,3 B$ & $62,6 \mathrm{~B}$ & $80,0 \mathrm{~B}$ & $114,0 \mathrm{~B}$ & $96,6 \mathrm{~B}$ & 56,2 \\
\hline \multirow[t]{3}{*}{ Óxido } & $6,0 \mathrm{~A}$ & $15,0 \mathrm{AB}$ & $29,3 \mathrm{~B}$ & $46,6 \mathrm{~B}$ & $65,3 \mathrm{~A}$ & $83,3 \mathrm{~A}$ & $118,3 \mathrm{~A}$ & $98,6 \mathrm{AB}$ & 57,8 \\
\hline & \multicolumn{9}{|c|}{ LVtma - cerrado nativo } \\
\hline & 10 & 20 & 40 & 60 & 80 & 100 & 120 & 140 & Médias \\
\hline \multirow[t]{3}{*}{ Óxido } & $9,6 \mathrm{~A}$ & $18,0 \mathrm{~A}$ & $39,0 \mathrm{~A}$ & $57,3 \mathrm{~A}$ & $77,6 \mathrm{~A}$ & $95,6 \mathrm{~A}$ & $113,0 \mathrm{~A}$ & $128,0 \mathrm{~A}$ & 67,3 \\
\hline & \multicolumn{9}{|c|}{ LAtm - cultivo de milho } \\
\hline & 10 & 20 & 40 & 60 & 80 & 100 & 120 & 140 & Médias \\
\hline Sulfato & $6,7 \mathrm{~A}$ & $14,1 \mathrm{~A}$ & $30,8 \mathrm{~A}$ & $48,0 \mathrm{~A}$ & $65,7 \mathrm{~B}$ & $84,3 \mathrm{~B}$ & $100,3 B$ & $119,8 B$ & 58,7 \\
\hline EDTA $^{1}$ & $8,1 \mathrm{~A}$ & $17,1 \mathrm{~A}$ & $33,8 \mathrm{~A}$ & $52,5 \mathrm{~A}$ & $68,0 \mathrm{AB}$ & $82,5 \mathrm{~B}$ & $104,6 \mathrm{~B}$ & $115,1 \mathrm{~B}$ & 60,2 \\
\hline Óxido & $7,1 \mathrm{~A}$ & $16,1 \mathrm{~A}$ & $34,7 \mathrm{~A}$ & $53,4 \mathrm{~A}$ & $73,7 \mathrm{~A}$ & $91,7 \mathrm{~A}$ & $112,3 \mathrm{~A}$ & $132,0 \mathrm{~A}$ & 65,1 \\
\hline
\end{tabular}

'Quelato; *Valores seguidos de mesma letra na coluna não diferem entre si pelo teste de Tukey a $5 \%$ de significância.

fonte quelatada (Mn-IDHA e EDDHA) em solo com plantas de morango.

No LAtm - cultivo de milho, o teor de Mn foi superior com o uso do fertilizante óxido a partir da concentração de $80 \mathrm{mg}$ $\mathrm{dm}^{-3} \mathrm{em}$ relação as outras fontes, todavia, nas concentrações mais baixas não foi observada diferença significativa (Tabela 5). Resultados semelhantes foram encontrados por Benett et al. (2012), que observaram diferenças significativas com uso de fonte quelatada ou sulfatada, ao avaliarem diferentes fontes de Mn aplicados via solo, na qualidade tecnológica e produtividade de colmos de cana-de-açúcar (RB 86-7515) nos dois primeiros cortes.

Conforme a Figura 2, quando se comparou o comportamento das doses, independente do tipo de fonte o teor disponível de $\mathrm{Mn}$ aumentou linearmente conforme o aumento das doses.

Observa-se que ambas as fontes apresentaram o mesmo comportamento em relação ao aumento da dose. Apesar do aumento na disponibilidade de Mn com aumento das doses, não verificou acentuado aumento nos tratamentos com EDTA comparado com as demais fontes (Figura $2 \mathrm{~b}$ e c). Este resultado não era esperado, pois o revestimento com agentes quelatantes tende a aumentar a disponibilidade do nutriente no solo (López-Rayo et al., 2012; López-Rayo et al., 2013; Mclaughlin et al, 2013).

Esses resultados diferem dos obtidos por Matias (2010) que ao trabalhar em um Latossolo Amarelo de textura muito argilosa verificou maior disponibilização de $\mathrm{Mn}$ no solo com a fonte quelatada EDTA. A falta de resultados nos tratamentos para os teores de Mn, pode ser justificada pela faixa de $\mathrm{pH}$ próxima ou acima de 6 , que contribuem para a dissociação de $\mathrm{H}^{+}$de grupos $\mathrm{OH}^{-}$da matéria orgânica e dos óxidos de $\mathrm{Al}$ e Fe, aumentando a adsorção dos metais e subsequente precipitação, reduzindo a sua biodisponibilidade (Vieira, 2016).

Para os teores de Zn no solo, no LAtma - cultivo de soja e cultivo de milho maior disponibilidade foi apresentada com os tratamentos que receberam quelato de EDTA, todavia, não houve diferença significativa entre o sulfato e óxido na maioria das concentrações (Tabela 6), exceto na dose de $140 \mathrm{mg} \mathrm{dm}^{-3}$ como fertilizante na forma de óxido que apresentou menor teor de Zn. Não houve diferenças nos teores de Zn disponível entre
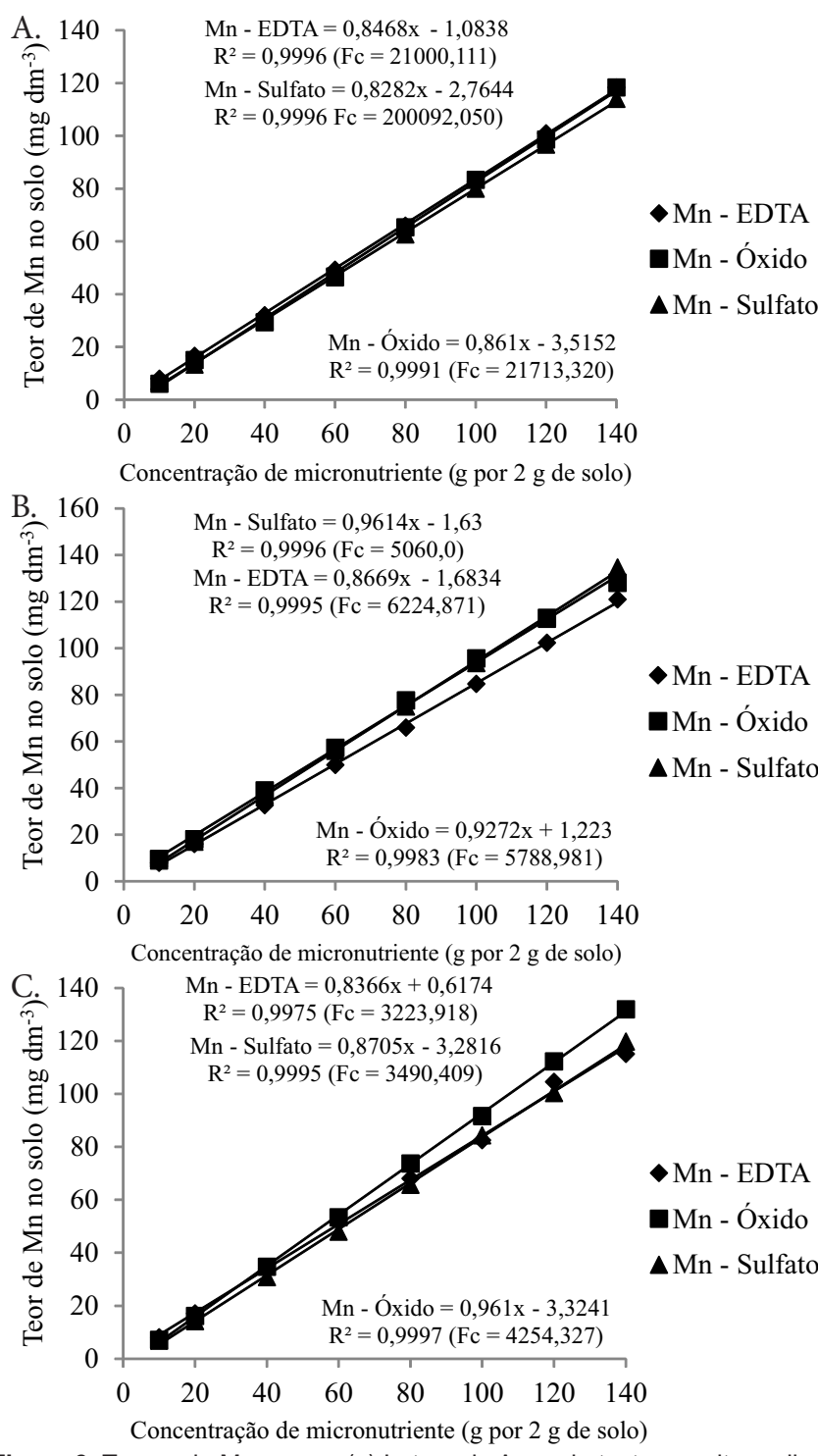

Figura 2. Teores de Mn em um (a) Latossolo Amarelo textura muito argilosa (LAtma - cultivo de soja), em um (b) Latossolo Vermelho de textura muito argilosa (LVtma - cerrado nativo), e em um (c) Latossolo Amarelo textura média (LAtm - cultivo de milho) em função de diferentes concentrações de Mn aplicados na forma de quelatos de EDTA, óxido e sulfato, Uberlândia, Minas Gerais. 
as fontes no LVtma - cerrado nativo e LAtm - cerrado nativo em chapadão, nas concentrações de 10 e $20 \mathrm{mg} \mathrm{dm}^{-3}$, porém, no LVtma - cerrado nativo a partir da concentração de $60 \mathrm{mg}$ $\mathrm{dm}^{-3}$ o teor disponível de $\mathrm{Zn}$ foi superior com o tratamento quelato de EDTA (Tabela 6).

Segundo Costa (2008) a eficiência das fontes de Zn depende da dose utilizada e conforme observado neste estudo a fonte EDTA apresentou maior disponibilidade de $\mathrm{Zn}$ em relação a fonte na forma de óxido nas maiores concentrações avaliadas. Além disso, os micronutrientes sofrem forte influência de $\mathrm{pH}$, sendo mais móveis em condições de pH baixo, ou seja, solos mais ácidos ( $\mathrm{pH}$ abaixo de 5,0 - 5,5), principalmente solos com elevado grau de intemperização (Latossolos), como é o caso das amostras de solos utilizadas no experimento (Vieira, 2016). Por isso, fontes que apresentam proteção ao elemento como o quelato, pode ser mais eficiente na disponibilização de micronutrientes (López-Rayo et al., 2012; Silva et al., 2014; Udeigwe et al., 2016). No LAtm - cerrado nativo em chapadão nas concentrações de 100 e $120 \mathrm{mgdm}^{-3}$ o teor de $\mathrm{Zn}$ disponível foi maior com uso de quelato de EDTA (Tabela 6).

No LAtm - cerrado nativo em chapadão, os teores de $\mathrm{Zn}$ com uso de ambas as fontes apresentaram o mesmo comportamento (Figura 3c). Mas, nos outros tipos de solo, o uso de quelato de EDTA favoreceu a maior disponibilidade de Zn (Figura 3a, b e c).

Resultados semelhantes foram observados por Nava et al. (2011), pela aplicação de fertilizantes formulados com diferentes fontes de Zn em um Latossolo Vermelho distroférrico cultivado com soja, onde verificaram aumento na disponibilidade de $\mathrm{Zn}$. Slaton et al. (2005) também constataram diferença nos teores de $\mathrm{Zn}$ no solo entre a aplicação da fonte orgânica (Zn-LS) e inorgânica $\left(\mathrm{ZnSO}_{4}\right)$. Avaliando a aplicação de diferentes fontes de $\mathrm{Zn}$ (sulfato, polifosfato e quelato de $\mathrm{Zn}$, respectivamente) na produtividade e acumulo de micronutrientes em plantas de milho, Beheraa et al. (2015), observaram maior absorção de $\mathrm{Zn}$ quando aplicou-se as fontes sulfatada e polifosfatada, consequência de sua maior disponibilização no solo.

Contudo, esses resultados diferem de Silva et al. (2014) que não verificaram diferença entre as fontes aplicadas (quelatadas e sulfatadas), somente aumento dos teores de $\mathrm{Zn}$ no solo em função do aumento das doses aplicadas. Assim como Costa (2008), em um Latossolo Vermelho distrófico, textura média, não constatou diferença nos teores de $\mathrm{Zn}$ no solo, com aplicação de sulfato de zinco, Zn-EDTA e Zn Lignosulfonato-LS.

Não se verificou grandes variações nos teores de $\mathrm{Zn}$ no solo, como observado no LVtma - cerrado nativo e o LAtm cerrado nativo em chapadão (Tabela 6 e Figura 3). De acordo Lopes et al. (2014), o Zn é um micronutriente que apresenta grande adsorção com os componentes da fase sólida do solo, por ligações físico-químicas cuja labilidade é dependente do ligante, como do conteúdo de minerais, óxidos e hidróxidos de $\mathrm{Fe}, \mathrm{Al}$ e $\mathrm{Mn}$, carbonatos e matéria orgânica, $\mathrm{pH}$ do solo (apresenta maior disponibilidade na faixa de $\mathrm{pH}$ 5,5 - 6,5), capacidade de troca de cátions (CTC) e da composição da matéria orgânica (Joris et al., 2012; Gonçalves et al., 2013; Brunetto et al., 2014; López-Rayo et al., 2012; Udeigwe et al., 2016; Vieira, 2016). Nesse sentido, a aplicação do Zn pela fonte na forma de óxido e sulfatada permitiu que o nutriente estivesse disponível no solo do mesmo modo que a fonte quelatada, em função das condições consideradas adequadas para disponibilidade do nutriente ( $\mathrm{pH}$, matéria orgânica e etc), assim como observado por Silva et al. (2014).

De modo geral, a eficiência dos fertilizantes quelatados aplicados ao solo pode ser de duas a cinco vezes maiores por unidade de micronutriente do que as fontes inorgânicas (López-Rayo et al., 2012), proporcionando maior estabilidade no solo (carbonatos, matéria orgânica e etc). Isso ocorre devido os agentes quelatantes regularem as reações do íon metálico pelo bloqueio dos sítios de reação destes íons, impedindo a sua entrada em reações químicas das quais participariam

Tabela 6. Teores de Zn disponíveis em um Latossolo Amarelo textura muito argilosa (LAtma - cultivo de soja), em um Latossolo Vermelho de textura muito argilosa (LVtma - cerrado nativo), em um Latossolo Amarelo de textura média (LAtm - cerrado nativo em chapadão), e em um Latossolo Amarelo textura média (LAtm - cultivo de milho) em função de diferentes concentrações de Zn aplicados na forma de quelatos de EDTA, óxido e sulfato, Uberlândia, Minas Gerais.

\begin{tabular}{|c|c|c|c|c|c|c|c|c|c|}
\hline \multicolumn{10}{|c|}{ Concentração $\left(\mathrm{mg} \mathrm{dm}^{-3}\right)$} \\
\hline \multirow{2}{*}{$\begin{array}{l}\text { Teores } \\
\text { de } \mathrm{Zn}^{*}\end{array}$} & \multicolumn{9}{|c|}{ LAtma - cultivo de soja } \\
\hline & 10 & 20 & 40 & 60 & 80 & 100 & 120 & 140 & Médias \\
\hline Sulfato & $1,0 \mathrm{~B}$ & $3,3 \mathrm{~B}$ & $11,0 \mathrm{~B}$ & $22,3 B$ & $34,0 \mathrm{~B}$ & $48,3 B$ & $62,3 \mathrm{~B}$ & $79,6 \mathrm{~B}$ & 32,7 \\
\hline EDTA $^{1}$ & $7,6 \mathrm{~A}$ & $14,6 \mathrm{~A}$ & $33,6 \mathrm{~A}$ & $51,3 \mathrm{~A}$ & $67,3 \mathrm{~A}$ & $84,6 \mathrm{~A}$ & $101,3 \mathrm{~A}$ & $117,3 \mathrm{~A}$ & 59,7 \\
\hline \multirow[t]{3}{*}{ Óxido } & $2,0 \mathrm{~B}$ & $5,0 \mathrm{~B}$ & $12,3 \mathrm{~B}$ & $22,6 \mathrm{~B}$ & $35,0 \mathrm{~B}$ & $48,0 \mathrm{~B}$ & $60,6 \mathrm{~B}$ & $75,6 \mathrm{C}$ & 32,6 \\
\hline & \multicolumn{9}{|c|}{ LVtma - cerrado nativo } \\
\hline & 10 & 20 & 40 & 60 & $\mathbf{8 0}$ & 100 & 120 & 140 & Médias \\
\hline Sulfato & $5,0 \mathrm{~A}$ & $11,0 \mathrm{~A}$ & $25,6 \mathrm{~A}$ & $42,0 \mathrm{~B}$ & $58,0 \mathrm{~B}$ & $76,0 \mathrm{~B}$ & $93,0 \mathrm{~B}$ & $112,6 \mathrm{~B}$ & 52,9 \\
\hline EDTA $^{1}$ & $7,0 \mathrm{~A}$ & $14,0 \mathrm{~A}$ & $32,0 \mathrm{~A}$ & $50,0 \mathrm{~A}$ & $66,6 \mathrm{~A}$ & $84,3 \mathrm{~A}$ & $104,0 \mathrm{~A}$ & $121,0 \mathrm{~A}$ & 59,9 \\
\hline Óxido & $5,0 \mathrm{~A}$ & $12,0 \mathrm{~A}$ & $25,0 \mathrm{~B}$ & $40,6 \mathrm{~B}$ & $58,3 \mathrm{~B}$ & $47,6 \mathrm{~B}$ & $90,3 \mathrm{~B}$ & $110,6 \mathrm{~B}$ & 52,0 \\
\hline \multicolumn{10}{|c|}{ LAtm - cerrado nativo em chapadão } \\
\hline & 10 & 20 & 40 & 60 & 80 & 100 & 120 & 140 & Médias \\
\hline EDTA $^{1}$ & $9,6 \mathrm{~A}$ & $17,0 \mathrm{~A}$ & $39,3 \mathrm{~A}$ & $58,0 \mathrm{~A}$ & $77,0 \mathrm{~A}$ & $97,0 \mathrm{~A}$ & $115,3 \mathrm{~A}$ & $129,3 \mathrm{~A}$ & 67,9 \\
\hline Óxido & $9,0 \mathrm{~A}$ & $16,6 \mathrm{~A}$ & $37,33 \mathrm{~A}$ & $56,0 \mathrm{~A}$ & $74,3 \mathrm{~A}$ & $93,3 \mathrm{~B}$ & $112,3 \mathrm{~B}$ & $132,0 \mathrm{~A}$ & 66,3 \\
\hline \multicolumn{10}{|c|}{ LAtm - cultivo de milho } \\
\hline & 10 & 20 & 40 & 60 & 80 & 100 & 120 & 140 & Médias \\
\hline Sulfato & $3,0 \mathrm{~B}$ & $8,3 \mathrm{~B}$ & $24,0 \mathrm{~B}$ & $41,6 \mathrm{~B}$ & $57,6 \mathrm{~B}$ & $76,6 \mathrm{~B}$ & $96,3 \mathrm{~B}$ & $115,3 \mathrm{~B}$ & 52,8 \\
\hline EDTA $^{1}$ & $9,0 \mathrm{~A}$ & $16,6 \mathrm{~A}$ & $37,3 \mathrm{~A}$ & $55,0 \mathrm{~A}$ & $73,3 \mathrm{~A}$ & $91,0 \mathrm{~A}$ & $111,3 \mathrm{~A}$ & $129,3 \mathrm{~A}$ & 65,3 \\
\hline Óxido & $2,0 \mathrm{~B}$ & $7,0 \mathrm{~B}$ & $18,3 \mathrm{C}$ & $32,6 \mathrm{C}$ & $48,0 \mathrm{C}$ & $65,0 \mathrm{C}$ & $81,6 \mathrm{C}$ & $96,0 \mathrm{C}$ & 43,8 \\
\hline
\end{tabular}

${ }^{1}$ Quelato; *Valores seguidos de mesma letra na coluna não diferem entre si pelo teste de Tukey a $5 \%$ de significância. 

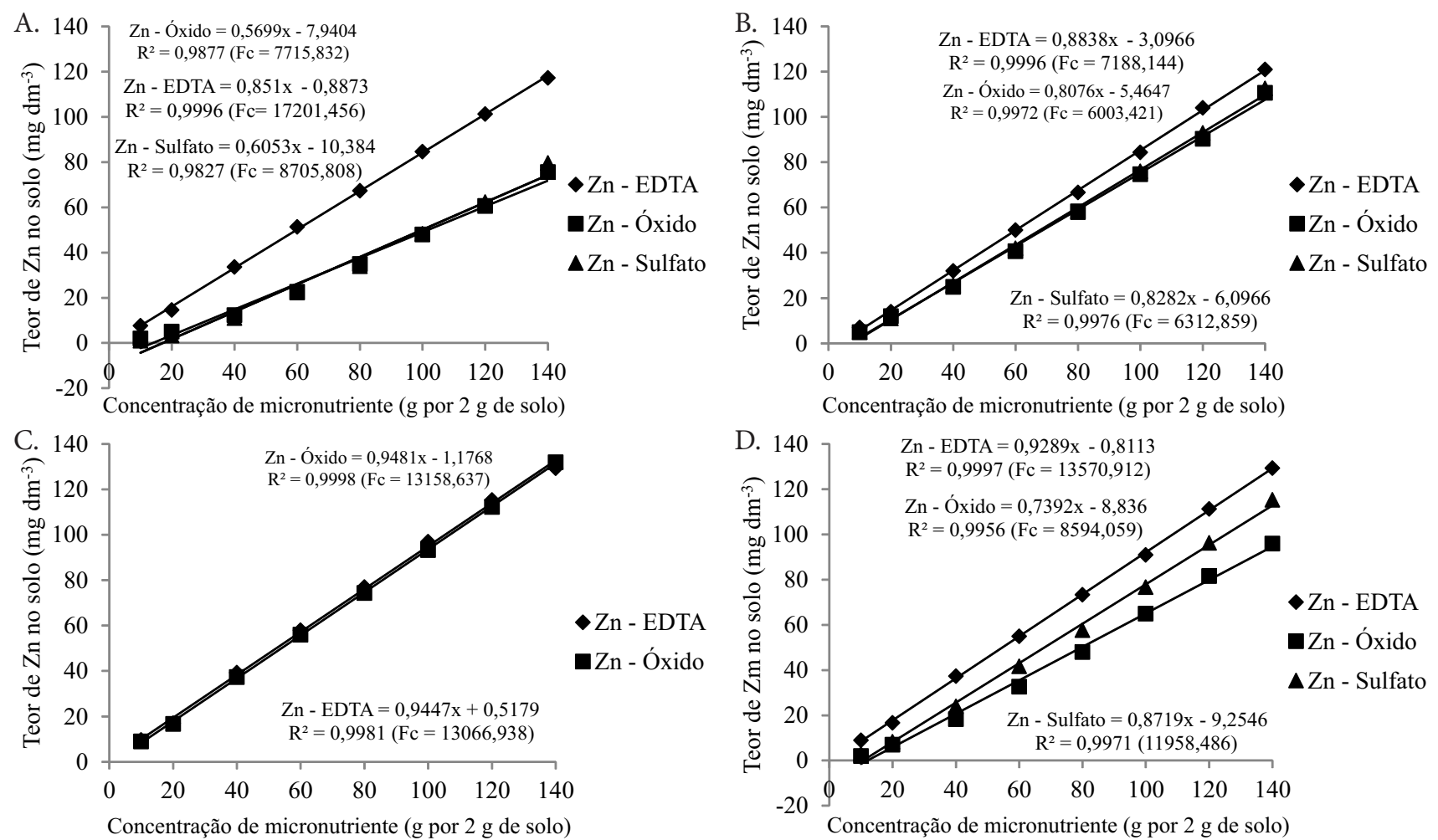

Figura 3. Teores de Zn em um (a) Latossolo Amarelo textura muito argilosa (LAtma - cultivo de soja), em um (b) Latossolo Vermelho de textura muito argilosa (LVtma - cerrado nativo), em um (c) Latossolo Amarelo de textura média (LAtm - cerrado nativo em chapadão), e em um (d) Latossolo Amarelo textura média (LAtm - cultivo de milho) em função de diferentes concentrações de Zn aplicados na forma de quelatos de EDTA, óxido e sulfato, Uberlândia, Minas Gerais.

normalmente e, além disso, são facilmente assimiláveis pelas plantas resultadas de sua estrutura orgânica (López-Rayo et al., 2012; Mclaughlin et al., 2013; Lopes et al., 2014; Udeigwe et al., 2016).

$\mathrm{O}$ uso de fertilizantes quelatados apresentam a vantagem de manterem os íons em uma forma solúvel, em ambientes onde os elementos poderiam ser precipitados, pelos hidróxidos, óxidos de ferro e alumínio, fosfatos, carbonatos e outros agentes químicos. No entanto, a eficiência de fertilizantes quelatados não depende somente do tipo de quelado, mas de outros fatores (solo-planta), como a capacidade dos agentes quelantes manterem-se solúveis na solução e habilidade da planta em assimilar os nutrientes (López-Rayo et al., 2012; Udeigwe et al., 2016).

\section{Conclusões}

O uso de fertilizante a base de quelato de EDTA proporciona aumento nos teores de $\mathrm{Cu}$ (cobre) e $\mathrm{Zn}$ (zinco) nos solos avaliados (LAtma - cultivo de soja; LVtma - cerrado nativo e LAtm - cultivo de milho).

A aplicação de fontes de micronutrientes (quelato de EDTA, sulfato e na forma de óxido) não proporciona diferenças na disponibilidade de Mn (manganês).

Nas amostras do Latossolo Vermelho de textura muito argilosa (LVtma - cerrado nativo) a disponibilidade de $\mathrm{Cu}$ aumenta linearmente em função da aplicação de fertilizante quelatado (EDTA), sendo mais acentuada do que nos demais solos (LAtm - cerrado nativo em chapadão e LAtm - cultivo de milho).
O fertilizante quelatado (EDTA) aumenta linearmente de maneira mais acentuada os teores de $\mathrm{Zn}$ nas amostras do solo Latossolo Amarelo textura muito argilosa e Latossolo Amarelo de textura média.

Em geral, a aplicação de fertilizante quelatado (EDTA) proporciona incrementos nos níveis de $\mathrm{Cu}$ e $\mathrm{Zn}$.

\section{Literatura Citada}

Alleoni, L.R.F.; Mello, J.W.V.; Rocha, W.D.S. Eletroquímica, adsorção e troca iônica no solo. In: Melo, V. F.; Alleoni, L. R. F. (Eds.). Química e mineralogia do solo: aplicações. Viçosa: SBCS, 2009. v.2, p.69-130.

Beheraa, S.K.; Shuklab, A.K.; Singhb, M.V.; Wanjarib, R.H., Singh, P. Yield and zinc, copper, manganese and iron concentration in maize (Zea mays 1.) grown on vertisol as influenced by zinc application from various zinc fertilizers. Journal of Plant Nutrition, v.38, n.10, p.1544-1557, 2015. https://doi.org/10.1080/01904167.2014.992537.

Benett, C.G.S.; Buzetti, S.; Silva, K.S.; Teixeira Filho, M.C.M.; Pariz, C.M.; Maeda, A.S.; Garcia, C.M.P. Qualidade e produtividade da cana planta e cana soca em função de doses e fontes de manganês. Bioscience Journal, v. 28, n. 2, p. 198-205, 2012. http://www.seer.ufu.br/index.php/ biosciencejournal/article/view/11680. 17 Mai. 2017.

Brunetto, B.; Schmitt, D.E.; Comin, J.J.; Miotto, A.; Moraes, M.P.; Heinzen, H. Frações de cobre e zinco em solos de vinhedos no Meio Oeste de Santa Catarina. Revista Brasileira de Engenharia Agrícola e Ambiental, v. 18, n. 8, p. 805-810, 2014. https://doi.org/10.1590/1807-1929/ agriambi.v18n08p805-810. 
Carvalho, V.G.; Nascimento, C.W.A.; Biondi, C.M. Potencial de fertilizantes e corretivos no aporte de micronutrientes ao solo. Revista Brasileira de Ciência do Solo, v. 36, n.3, p. 931-938, 2012. https://doi.org/10.1590/S010006832012000300023.

Costa, R. S.S. Aplicação de quelatos de zinco em um solo deficiente cultivado com milho em casa de vegetação. Jaboticabal: Universidade Estadual Paulista, Faculdade de Ciências Agrárias e Veterinárias, 2008. 31p. Dissertação Mestrado. http://hdl.handle.net/11449/96935. 10 Jan. 2016.

Empresa Brasileira de Pesquisa Agropecuária - Embrapa. Sistema brasileiro de classificação de solos. 3.ed. Brasília: Embrapa, 2013. 353 p.

Empresa Brasileira de Pesquisa Agropecuária - Embrapa. Manual de análises químicas de solos, plantas e fertilizantes. 2. ed. Brasília: Embrapa Informação Tecnológica, 2009. 627p.

Ferreira, D.F. SISVAR: um programa para análises e ensino de estatística. Revista Symposium, v.6, n.2, p.36-41, 2008. http://www.dex.ufla.br/ danielff/meusarquivospdf/art63. pdf. 29 Dez. 2015.

Gonçalves, M.S.; Bettin, J.P.; Silva Junior, L.C.S.; Sampaio, S.C.; Dal Bosco, T.C. Adequação dos modelos de langmuir e freundlich na adsorção de cobre em soloargiloso do sul do Brasil. HOLOS, v. 29 , n. 4, p. 37-44, 2013. https://doi. org/10.15628/holos.2013.1458.

Jalali, M.; Latifi, Z. Measuring and simulating effect of organic residues on the transport of cadmium, nickel, and zinc in a calcareous soil. Journal of Geochemical Exploration, in press, 2017. https://doi.org/10.1016/j.gexplo.2017.05.001.

Jalali, M.; Moradi, F. Competitive sorption of $\mathrm{Cd}, \mathrm{Cu}, \mathrm{Mn}$, $\mathrm{Ni}, \mathrm{Pb}$ and $\mathrm{Zn}$ in polluted and unpolluted calcareous soils. Environmental Monitoring and Assessment, v. 183, n. 11, p. 8831-8846, 2013. https://doi.org/10.1007/s10661-0133216-1.

Joris, H.A.W.; Fonseca, A.F.; Asami, V.Y.; Briedis, C.; Borszowskei, P.R.; Garbuio, F.J. Adsorção de metais pesados após calagem superficial em um Latossolo Vermelho sob sistema de plantio direto. Revista Ciência Agronômica, v. 43, n. 1, p. 1-10, 2012. https://doi. org/10.1590/S1806-66902012000100001.

Li, T.; Tao, Q.; Liang, C.; Shohag, M. J. I.; Yang, X.; Sparks, D. L. Complexation with dissolved organic matter and mobility control of heavy metals in the rhizosphere of hyperaccumulator Sedum alfredii. Environmental Pollution, v. 182 , p. $248-255,2013$. https://doi.org/10.1016/j. envpol.2013.07.025.

Lopes, C.; Campos, M. L.; Silveira, C. B.; Gatiboni, L. C.; Miquelutti, D. J.; Cassol, P. C.; Medeiros, Í. F. Adsorção de $\mathrm{Cu}$ e $\mathrm{Zn}$ num Latossolo Vermelho tratado com dejetos suínos. Revista Ceres, v. 61, n. 6, p. 997-1005, 2014. https:// doi.org/10.1590/0034-737X201461060016.

López-Rayo, S.; Correas, C.; Lucena, J.J. Novel chelating agents as manganese and zinc fertilisers: characterisation, theoretical speciation and stability in solution. Chemical Speciation and Bioavailability, v. 24, n.3, p. 147-158, 2012. https://doi.org/10.3184/095422912X13409631969915.
López-Rayo, S.; Nadal, P.; Pozo, M.A.; Domínguez, A.; Lucena, J. Efficacy of micronutrient chelate treatments in commercial crop of strawberry on sand culture. Communications in Soil Science and Plant Analysis, v. 44, n.1-4, p. 826-836, 2013. https://doi.org/10.1080/00103624 .2013 .749440 .

Matias, F.I.; Silva, T.S.; Vasconcelos, A.C.P.; Silva, A.A.; Lana, R.M.Q.; Junior, P.A.C. Adsorção e disponibilidade de zinco decorrente da utilização de: quelatado com EDTA, fertilizante liquido e sulfato. In: Simpósio Mineiro de Ciências do Solo, 1., 2010, Viçosa. Anais.Viçosa: Universidade Federal de Viçosa, 2010. p.1-3.

Mclaughlin, M.; Stacey, S.; Lombi, E. Chelating agents for micronutrient fertilisers. US Pat. 8,426,338, abr. 2013. 12p. https://www.google.com/patents/ EP1910250B1?cl=en\&hl=pt-BR. 17 May. 2017.

Nava, I.A.; Junior, A.C.G.; Nacke, H.; Guerini, V.L.; Schwante, D. Disponibilidade dos metais pesados tóxicos cádmio, chumbo e cromo no solo e tecido foliar da soja adubada com diferentes fontes de NPK $+Z n$. Ciência e Agrotecnologia, v.35, n.5, p. 884-892, 2011. https://doi.org/10.1590/S141370542011000500004

Peak, D.; Ford, R.G.; Sparks, D.L. An in situ ATR-FTIR investigation of sulfate bonding mechanisms on goethite. Journal of Colloid and Interface Science, v.218, n.1, p.289299, 1999. https://doi.org/10.1006/jcis.1999.6405.

Raij, B.V. Fertilidade do solo e manejo de nutrientes. Piracicaba: International Plant Nutrition Institute, 2011. 420p.

Silva, A.A.; Couto Junior, P.A.; Lana, A.M.Q.; Lana, R.M.Q. Teores de micronutrientes no solo e foliar com aplicação de fontes quelatadas e sulfatadas em feijão. Engenharia Agrícola, v.34, n.1, p.28-37, 2014. https://doi.org/10.1590/ S0100-69162014000100004.

Slaton, N.A.; Gbur Junior, E.E.; Wilson Junior, C.E.; Norman, R.J. Rice response to granular zinc sources varying in watersoluble zinc. Soil Science Society of America Journal, v.69, n.2, p. 443-454, 2005. https://doi.org/10.2136/ sssaj2005.0443.

Soares, M. R. Coeficiente de distribuição (Kd) de metais pesados em solos do Estado de São Paulo. Piracicaba: ESALQ, 2004. 202p. Dissertação Mestrado. https://doi. org/10.11606/T.11.2005.tde-31052005-170719.

Udeigwe, T.K.; Eichmann, M.B.; Menkiti, M.C. Examining the fixation kinetics of chelated and non-chelated copper micronutrient and the applications to micronutrient management in semi-arid alkaline soils. Solid Earth, v. 7, p. 311-321, 2016. https://doi.org/10.5194/se-7-311-2016.

Vieira, J.S. Disponibilidade e variabilidade espacial de cobre, ferro, manganês e zinco nos solos do sertão central do Ceará. Teresina: Universidade Federal do Piauí, 2016. 46p. Dissertação Mestrado. http://repositorio.ufpi.br/xmlui/ handle/123456789/346. 10 Jan. 2016. 